\title{
Charge-Ordering and Magnetoelastic Coupling Effects on the Magnetocaloric Properties of Manganites
}

\author{
M.S. Reis ${ }^{a, b}$, A.M. Gomes ${ }^{b}$, J.P. AraúJo ${ }^{c}$, P.B. TAVAreS ${ }^{d}$, \\ J.S. Amaral ${ }^{a}$, I.S. Oliveira ${ }^{b}$ AND V.S. Amaral ${ }^{a}$ \\ ${ }^{a}$ Departamento de Física and CICECO, Universidade de Aveiro \\ 3810-193 Aveiro, Portugal \\ ${ }^{b}$ Centro Brasileiro de Pesquisas Físicas, \\ R. Dr. Xavier Sigaud 150, 22290-180 Rio de Janeiro-RJ, Brasil \\ ${ }^{c}$ Departamento de Física and IFIMUP, Universidade do Porto \\ 4169-007 Porto, Portugal \\ ${ }^{d}$ Departamento e Centro de Química \\ Universidade de Trás-os-Montes e Alto Douro, 5001-911 Vila Real, Portugal
}

A study of the magnetocaloric properties of $\mathrm{La}_{1-x-y} \mathrm{Y}_{y} \mathrm{Ca}_{x} \mathrm{MnO}_{3}$, $\mathrm{La}_{1-x} \mathrm{Cd}_{x} \mathrm{MnO}_{3}$, and $\mathrm{Pr}_{1-x} \mathrm{Ca}_{x} \mathrm{MnO}_{3}$ manganites with ferromagnetic and charge-ordered states is presented. The ferromagnetic lanthanum manganites show a negative entropy change peak $\Delta S$ under the application of a magnetic field at the Curie temperature. However, the magnetoelastic coupling effects lead to a first-order paramagnetic-ferromagnetic transition and an increased $\Delta S$ compared to simple ferromagnets. This effect is analyzed in the framework of the Landau theory of phase transitions which shows a comparable influence of the magnetoelastic couplings and the ordinary magnetic ordering effect. The $\operatorname{Pr}_{1-x} \mathrm{Ca}_{x} \mathrm{MnO}_{3}$ system shows a rich electric and magnetic phase diagram, with a ferromagnetic insulator phase for $0.15<x<0.30$ and coexisting charge-ordering and antiferromagnetic insulator behavior for $0.30<x<0.85$. The magnetocaloric effect results for $x=0.25$ and 0.30 show a simple ferromagnetic phase transition effect. For the samples above the onset concentration for the charge ordering $(\approx 0.30)$ an anomalous magnetic entropy change is observed below the charge-ordering temperature $\left(T_{\mathrm{co}}\right)$. This effect is associated with a positive contribution from the magnetic entropy change due to charge ordering, superimposed to the negative contribution due to spin ordering.

PACS numbers: 75.30.Sg, 75.30.Kz, 75.80.+q 


\section{Introduction}

In a typical paramagnetic or ferromagnetic material, the application of a magnetic field leads to an alignment of the magnetic moments. Starting from a zero magnetic field state with randomly oriented magnetic moments, the increase in the magnetic field leads to a decrease in the magnetic part of the entropy $\left(S_{M}\right)$, as this thermodynamic function is associated with the degree of magnetic order. If this process is performed adiabatically, without heat transfer to the exterior, the material temperature will increase, as the lattice entropy increases. When the magnetic field is turned off, randomizing again the magnetic moments, the opposite situation occurs, and the material temperature decreases. This adiabatic temperature change induced by a magnetic field variation is called magnetocaloric effect (MCE). A magnetic cycle refrigeration system is obtained by allowing the magnetic material to expel heat to an exterior heat sink after application of the magnetic field, reduce the field adiabatically and subsequently extract heat from the system to be cooled. This technique has been used for many years to obtain ultra-low temperatures by adiabatic demagnetization cooling and more recently, has found application in cryogenic engineering to raise the efficiency of gas liquefaction systems. In general, considerable MCE effects are expected in connection with the large entropy change associated with the ferromagnetic to paramagnetic phase transition in the vicinity of the Curie temperature $T_{\mathrm{c}}$. In recent years the interest on the research of magnetocaloric cooling systems starting from room temperature has raised considerably due to its potential impact on environmental concerns and energy saving. In fact, this technology does not require any ozone-depleting nor hazardous chemicals and the thermal efficiency is higher than gas-compression refrigerators. Gadolinium is a good candidate for magnetic refrigeration systems near room temperature $\left(T_{\mathrm{c}}=294 \mathrm{~K}\right)$, with $2.8 \mathrm{~J} /(\mathrm{kg} \mathrm{K})$ and $2.6 \mathrm{~K}$ of magnetic entropy and temperature changes, respectively, under $10 \mathrm{kOe}$ of magnetic field variation [1]. In the last decade, an increased research effort on magnetocaloric studies has revealed new materials with large magnetic entropy changes under an applied magnetic field near magnetic phase transitions, such as $\mathrm{Gd}_{5}\left(\mathrm{Si}_{2-x} \mathrm{Ge}_{2+x}\right)$ [2], $\mathrm{MnFeP}{ }_{1-x} \mathrm{As}_{x}$ [3], $\mathrm{LaFe}_{13-x} \mathrm{Si}_{x}$ [4], and manganites $\mathrm{R}_{1-x} \mathrm{D}_{x} \mathrm{MnO}_{3}(\mathrm{R}=$ rare-earth $\mathrm{La}, \mathrm{Pr}$ and $\mathrm{D}=$ divalent $\mathrm{Ca}, \mathrm{Sr}$ ) [5]. One of the most interesting features of these new materials is the possibility of tuning the operation range (the Curie temperature) by a suitable chemical composition adjustment. In this work we report magnetocaloric studies on some polycrystalline samples of the manganite systems $\mathrm{La}_{1-x-y} \mathrm{Y}_{y} \mathrm{Ca}_{x} \mathrm{MnO}_{3}, \mathrm{La}_{1-x} \mathrm{Cd}_{x} \mathrm{MnO}_{3}$, and $\mathrm{Pr}_{1-x} \mathrm{Ca}_{x} \mathrm{MnO}_{3}$, with Mn mixed valence ions $\left(\mathrm{Mn}^{4+}, \mathrm{Mn}^{3+}\right)$ presenting ferromagnetic and charge-ordered states due to the interplay between charge, spin, and lattice degrees of freedom $[6,7]$.

Experimentally, a convenient procedure for the search of suitable materials for MCE applications is the determination of the magnetic entropy change $\Delta S_{\mathrm{M}}(T, \Delta H)$, which is a function of the temperature and the magnetic field change $\Delta H=H_{\mathrm{F}}-H_{\mathrm{I}}$. A typical representation is the entropy change with $H_{\mathrm{I}}=0$, 
as a function of $T$, with constant $\Delta H=H_{\mathrm{F}}$. The magnetic entropy dependence with the magnetic field $H$ is related to the magnetization $M$ via the thermodynamic Maxwell relation [8]:

$$
\left(\frac{\partial S_{\mathrm{M}}(T, H)}{\partial H}\right)_{T}=\left(\frac{\partial M(T, H)}{\partial T}\right)_{H},
$$

which after integration yields

$$
\Delta S_{\mathrm{M}}(T)_{\Delta H}=\int_{H_{\mathrm{I}}}^{H_{\mathrm{F}}} \mathrm{d} S_{\mathrm{M}}(T, H)_{T}=\int_{H_{\mathrm{I}}}^{H_{\mathrm{F}}}\left(\frac{\partial M(T, H)}{\partial T}\right)_{H} \mathrm{~d} H .
$$

Hence $\Delta S_{\mathrm{M}}(T, \Delta H)$ can be determined from magnetization measurements, more conveniently than from calorimetric measurements. Generally, since temperature stabilization is the longest step in the process of magnetization data collection, the measurements are usually carried out isothermically by varying the magnetic field, for a range of temperature values. Suitable numerical procedures are then used for derivation with respect to temperature of interpolated $M(T, H)$ data points and final magnetic field integration.

\section{Experimental}

Manganite samples were prepared by the ceramic route, starting from stoichiometric amounts of $\mathrm{Pr}_{2} \mathrm{O}_{3}, \mathrm{La}_{2} \mathrm{O}_{3}, \mathrm{Y}_{2} \mathrm{O}_{3}, \mathrm{CaCO}_{3}, \mathrm{CdCO}_{3}$, and $\mathrm{MnO}_{2}$, and heating in air, with 3-5 intermediate crushing/pressing steps until all raw materials reacted to form perovskite. The La-manganite powders were sintered in air at $1300^{\circ} \mathrm{C}$ for $50 \mathrm{~h}$ and slowly cooled to room temperature. The Pr-manganites were sintered in air at $1350^{\circ} \mathrm{C}$ for $45 \mathrm{~h}$, with a subsequent fast freezing of the samples. The LaCd-manganite sample required lower sintering temperatures [9]. The samples studied have compositions $\mathrm{La}_{0.67} \mathrm{Cd}_{0.25} \mathrm{MnO}_{3}(\mathrm{LaCd}), \mathrm{La}_{0.60} \mathrm{Y}_{0.07} \mathrm{Ca}_{0.33} \mathrm{MnO}_{3}$ (LaYCa) and $\operatorname{Pr}_{1-x} \mathrm{Ca}_{x} \mathrm{MnO}_{3}(x=0.25,0.30,0.32,0.35$, and 0.40). From X-ray diffraction we find that all the samples are pure phase. The temperature and magnetic field dependences of the magnetization $M(T, H)$ were determined using a commercial superconducting quantum interference device (SQUID) magnetometer (Quantum Design).

\section{Magnetoelastic effects contribution}

The studied $\mathrm{La}_{0.60} \mathrm{Y}_{0.07} \mathrm{Ca}_{0.33} \mathrm{MnO}_{3}$ and $\mathrm{La}_{0.67} \mathrm{Cd}_{0.33} \mathrm{MnO}_{3}$ manganites are both ferromagnetic with very close Curie temperatures, $T_{c} \approx 150 \mathrm{~K}$. However, their electric properties are very different: the LaYCa sample is metallic below $T_{c}$ and presents large magnetoresistance, while the $\mathrm{LaCd}$ sample is insulating with small magnetoresistance [9]. In addition, the LaCd sample does not show any anomaly in the thermal expansion at the transition and its magnetostriction is very small, in strong contrast to the LaYCa sample [10]. These contrasting properties were 
interpreted [10-12] using the Landau theory of phase transitions [13] and related to the additional contribution of magnetoelastic couplings and electron condensation energy (metal-insulator transition) on the coefficient $B$ in the Landau theory magnetic energy expansion [14]:

$$
G(T, M)=G_{0}(T)+\frac{1}{2} A M^{2}+\frac{1}{4} B M^{4}+\frac{1}{6} C M^{6}-M H .
$$

To account for 1st (for $B<0$ ) and 2nd order (for $B>0$ ) phase transitions the expansion includes terms up to the 6 th power in $M$. The coefficients $A, B$, and $C$ depend on temperature. The additional interactions decrease $B$ from the regular positive values found in normal ferromagnets to negative values, changing the order of the phase transition.

From energy minimization, a magnetic equation of state is derived within this theory

$$
\frac{H}{M}=A+B M^{2}+C M^{4},
$$

which leads directly to the well known Arrott plots and the corresponding magnetic entropy is obtained from differentiation of the magnetic part of the free energy (Eq. (3)) with respect to temperature

$$
S_{\mathrm{M}}(T, H)=-\left(\frac{\partial G}{\partial T}\right)_{H}=-\frac{1}{2} A^{\prime}(T) M^{2}-\frac{1}{4} B^{\prime}(T) M^{4}-\frac{1}{6} C^{\prime}(T) M^{6} .
$$

$A^{\prime}(T), B^{\prime}(T)$, and $C^{\prime}(T)$ are the temperature derivatives of the expansion coefficients. This relation between magnetic entropy $S_{\mathrm{M}}(T, H)$ and magnetization could also be obtained using Eq. (2) and the equation of state (Eq. (4)), noting that within the Landau theory $S_{\mathrm{M}}=0$ for $M=0$.

For both samples, the expansion coefficients were determined by fitting the measured magnetization to the equation of state (Eq. (4)) [10-12]. For the LaYCa
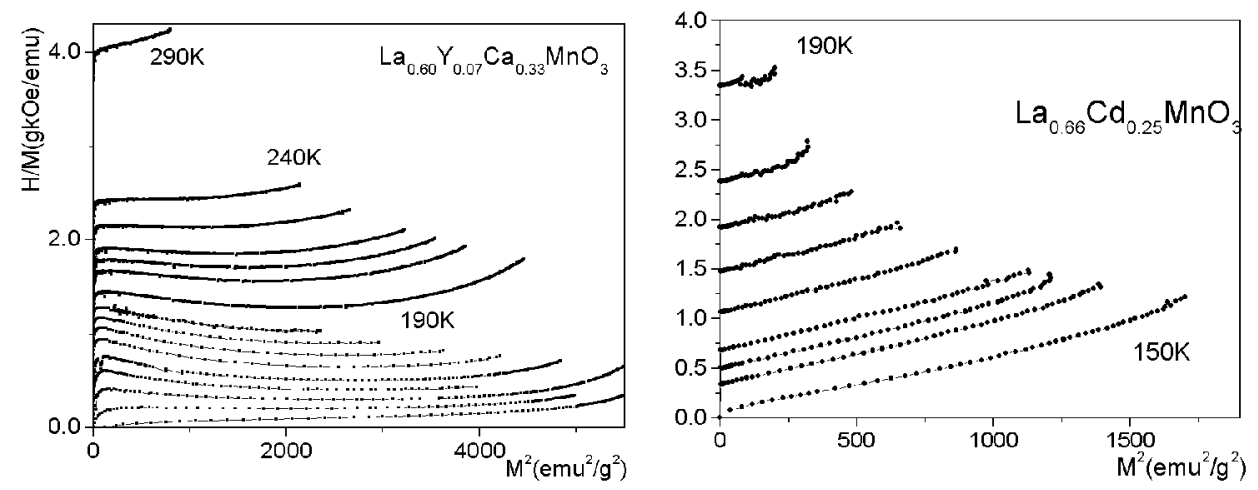

Fig. 1. Arrott plots for the samples $\mathrm{La}_{0.60} \mathrm{Y}_{0.07} \mathrm{Ca}_{0.33} \mathrm{MnO}_{3}$ (left) and $\mathrm{La}_{0.66} \mathrm{Cd}_{0.25} \mathrm{MnO}_{3}$ (right) in the paramagnetic phase. 

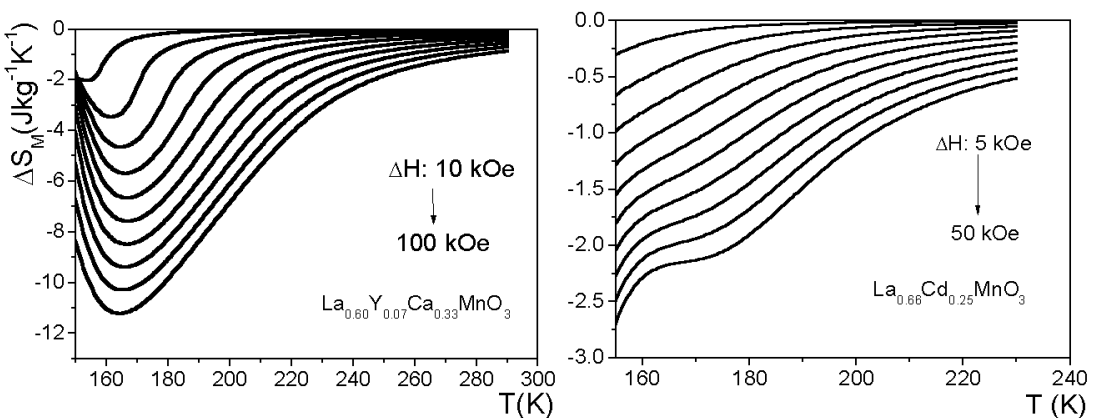

Fig. 2. Variation of magnetic entropy, $\Delta S_{\mathbb{M}}(T, H)$ for different values of applied magnetic field for samples $\mathrm{La}_{0.60} \mathrm{Y}_{0.07} \mathrm{Ca}_{0.33} \mathrm{MnO}_{3}$ (left) and $\mathrm{La}_{0.66} \mathrm{Cd}_{0.25} \mathrm{MnO}_{3}$ (right).
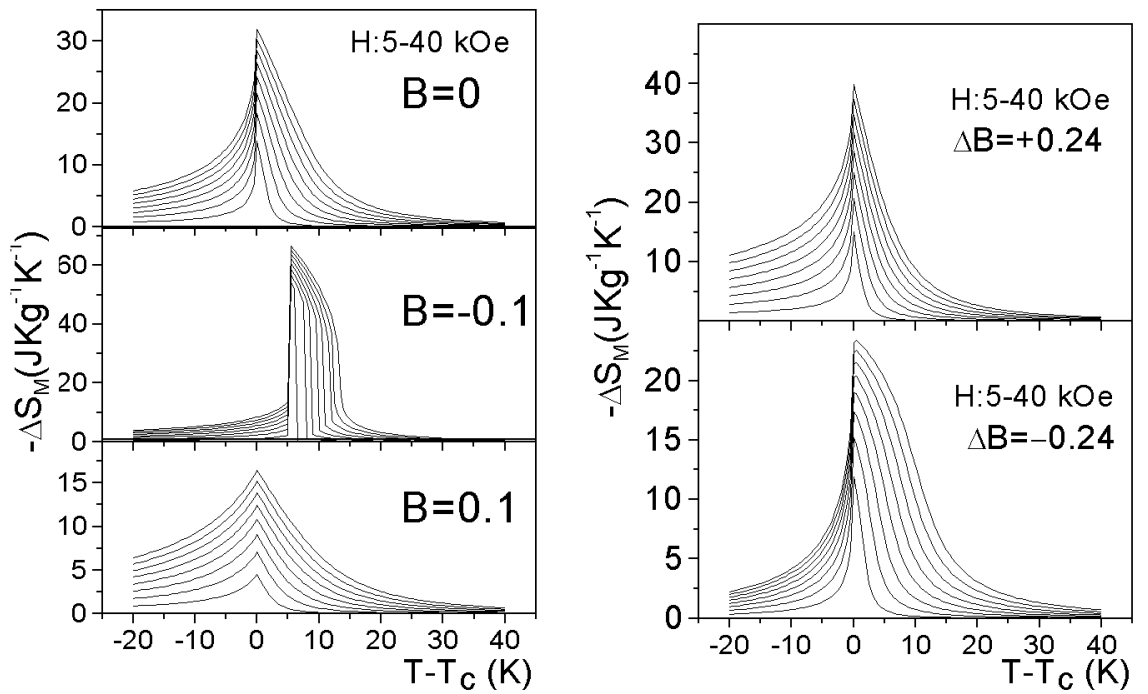

Fig. 3. Modeling of the magnetic entropy $S_{\mathrm{M}}(T, H)$ using the Landau expansion near the phase transition. Left: $\Delta S_{\mathrm{M}}$ temperature dependence for constant $B$ coefficient. Right: $\Delta S_{\mathrm{M}}$ temperature dependence for positive and negative derivative of $B$ over temperature. $\Delta B$ is the variation in the temperature interval displayed.

sample, $B$ is negative with a minimum at $200 \mathrm{~K}, 50 \mathrm{~K}$ above $T_{\mathrm{c}}$. In contrast, for the LaCd sample, $B$ is positive and almost constant. The corresponding $\mathrm{Ar}$ rott plots with representative data in the paramagnetic phase range are shown in Fig. 1. Using the temperature dependence of the coefficients the magnetic entropy $\Delta S_{\mathrm{M}}(T, H)$ was calculated and is shown in Fig. 2. A larger magnetic entropy change is obtained in the LaYCa sample, with a well defined maximum above $T_{\mathrm{c}}$. The analysis of the contributions in Eq. (5) shows that this results from the larger magnetoelastic coupling and the first-order transition effects. In fact, for a simple 
ferromagnet, with $B$ and $C$ constant and positive, $\Delta S_{\mathrm{M}}(T, H)$ would present a narrow peak at $T_{c}$. To clarify the effect of the additional interactions on the magnetic entropy through the $B$ coefficient $S_{\mathrm{M}}(T, H)$ was modeled using the Landau expansion. Representative results are shown in Fig. 3 for constant $B$ (left) or constant $B^{\prime}$ (in the case with $B=0$ at $T_{\mathrm{c}}$ ) (right). $A(T)$ was taken linear vs. $T-T_{\mathrm{c}}$, with slope $67 \mathrm{gOe} / \mathrm{emu}$. $C$ was made constant: $5 \times 10^{-6} \mathrm{~g}^{5} \mathrm{Oe} / \mathrm{emu}^{5}$. $B$ values shown are in $\mathrm{g}^{3} \mathrm{Oe} / \mathrm{emu}^{3}$. The direct effect of $B^{\prime}$ on $\Delta S_{\mathrm{M}}$ is evidenced in Fig. 3 (right): positive $B^{\prime}$ leads to higher $\Delta S_{\mathrm{M}}$ peak values. These dependences (particularly when $B$ is negative above $T_{\mathrm{c}}$ ), are in close agreement with experimental results in many systems besides manganites, where a broad region of fairly high values is found: $\mathrm{RCO}_{2}$ [15] and LaFeSi metamagnets [4, 16], MnAs based systems [3]. Additionally, we mention that the Landau equation of state (4) provides a convenient numerical interpolation scheme to treat magnetization data in order to determine the entropy change.

\section{Charge-order contribution}

The $\operatorname{Pr}_{1-x} \mathrm{Ca}_{x} \mathrm{MnO}_{3}$ system shows a rich electric and magnetic phase diagram [17, 18]. At high temperatures the system is paramagnetic and insulator (PMI). For $x<0.15$ a canted magnetic insulator (CI) phase arises, whereas for $0.15<x<0.30$ a ferromagnetic insulating (FMI) phase is established. A more complex electric-magnetic phase diagram is found for $0.30<x<0.85$, where the charge-ordering (CO) effect coexists with an antiferromagnetic insulator (AFMI). The charge-ordered state is characterized by the existence of a regular array of $\mathrm{Mn}^{4+}$ and $\mathrm{Mn}^{3+}$ ions in the lattice. In addition, this insulating state can be switched to metallic by an applied magnetic field.

The isothermal magnetization $M(T, H)$ data were processed according to Eq. (2) to determine the magnetic entropy change $\Delta S_{\mathrm{M}}(T, \Delta H)$ as a function of temperature, for a magnetic field change $\Delta H=H_{\mathrm{F}}-H_{\mathrm{I}}$. The magnetic entropy change $\Delta S_{\mathrm{M}}$ for the magnetic field interval 0 to $40 \mathrm{kOe}$ in the temperature range $50-300 \mathrm{~K}$ is presented in Fig. 4. The results for $x \leq 0.30$ show a regular ferromagnetic phase transition effect with a negative peak at $T_{\mathrm{c}} \approx 130 \mathrm{~K}$. However, for the samples above the onset concentration for the charge ordering $(\approx 0.30)$ an anomalous magnetic entropy change is observed just below the charge-ordering temperature $\left(T_{\mathrm{co}}>200 \mathrm{~K}\right)$. A positive $\Delta S_{\mathrm{M}}$ peak develops, increasing in amplitude with $\mathrm{Ca}$ content (higher $T_{\mathrm{co}}$ ). This increase in the magnetic entropy is associated with the progressive suppression of charge ordering, leading to an increase in accessible states due to the enhancement of electron mobility, under an applied magnetic field. Interestingly, this effect is superimposed to the negative contribution due to spin ordering in the paramagnetic phase: above $T_{\mathrm{Co}}, \Delta S_{\mathrm{M}}$ is very similar for all the samples, and the charge-ordering effect leads to the positive contribution onset. Thus, considering that the spin contribution to the total magnetic entropy change of $x>0.30$ is almost similar to the purely spin contribution 


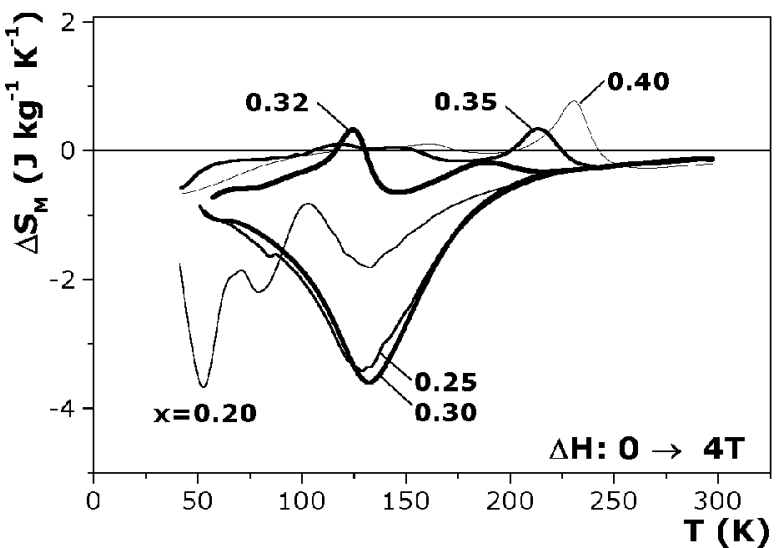

Fig. 4. Measured magnetic entropy change for the studied $\operatorname{Pr}_{1-x} \mathrm{Ca}_{x} \mathrm{MnO}_{3}$ samples with $H=40 \mathrm{kOe}$. The FMI samples show the usual negative contribution, whereas for the CO samples this phase transition is signalled by an increase in the magnetic entropy on cooling.

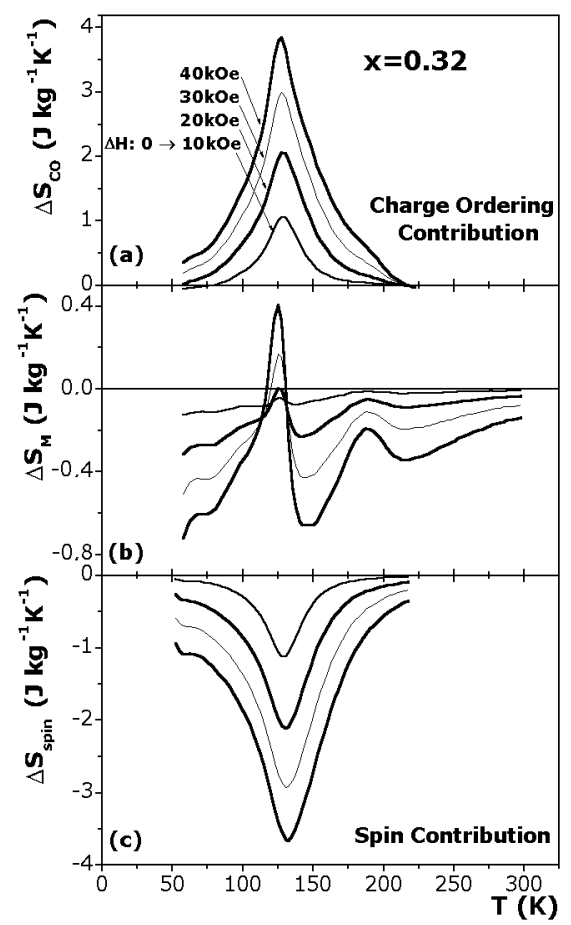

Fig. 5. For sample $\operatorname{Pr}_{1-x} \mathrm{Ca}_{x} \mathrm{MnO}_{3}$ where $x=0.32$ : temperature dependence of the (a) charge-ordering $\Delta S_{\text {co }}$ and (c) spin, $\Delta S_{\text {spin }}$, contributions, (b) total magnetic entropy change $\Delta S_{\mathrm{M}}$ under several values of field. 
of $x=0.30$, shifted to its $T_{\mathrm{N}}$, we can satisfactorily estimate the charge-ordering contribution. This separation of the $\mathrm{CO}$ and spin contribution to the total magnetic entropy change is shown in Fig. 5 for $x=0.32$, for several magnetic field values.

The behavior of the positive charge-ordering contribution, that peaks at $T_{\mathrm{N}}$, can be understood as follows. For $T_{\mathrm{N}}<T<T_{\mathrm{co}}$, i.e., still in the paramagnetic phase, the applied magnetic field force a rude alignment of the spins, increasing the $\mathrm{Mn}^{3+}-\mathrm{Mn}^{4+}$ electron hopping and decreasing the concentration of $\mathrm{Mn}^{3+}{ }_{-} \mathrm{Mn}^{4+}$ charge-ordered, when compared with the zero field case. Consequently, the entropy due the $\mathrm{CO}$ increase under an external applied magnetic field, allowing a positive CO entropy change. However, for temperatures immediately below $T_{\mathrm{N}}$, the applied magnetic field favors the increase in the antiferromagnetic spin arrangement, comparing to the case without field, and, consequently, the decrease in the $\mathrm{Mn}^{3+}-\mathrm{Mn}^{4+}$ electron mobility. Thus, the concentration of $\mathrm{Mn}^{3+}-\mathrm{Mn}^{4+}$ charge-ordered increases, implying in the decrease in the entropy change due the charge order, under an applied magnetic field.

The positive contribution of the charge ordering to the magnetic entropy has been reported in some manganite materials $[19,20]$ for which the CO antiferromagnetic insulator phase appears at lower temperatures than a ferromagnetic metallic phase, and the positive entropy peak signals this order-order transition. In our case, in contrast the $\mathrm{CO}$ effect manifests in a order-disorder transition, and the positive peak can be shifted to much below $T_{\mathrm{co}}$.

\section{Conclusions}

The present work reports studies of the magnetocaloric properties of $\mathrm{La}_{1-x-y} \mathrm{Y}_{y} \mathrm{Ca}_{x} \mathrm{MnO}_{3}, \mathrm{La}_{1-x} \mathrm{Cd}_{x} \mathrm{MnO}_{3}$, and $\mathrm{Pr}_{1-x} \mathrm{Ca}_{x} \mathrm{MnO}_{3}$ manganites with ferromagnetic and charge-ordered states. The lanthanum manganites show a negative entropy change peak $\Delta S$ under the application of a magnetic field at the Curie temperature. The magnetoelastic coupling effects which lead to a first-order paramagnetic-ferromagnetic transition and an increased $\Delta S$ compared to simple ferromagnets are analysed in the framework of the Landau theory of phase transitions. In the $\operatorname{Pr}_{1-x} \mathrm{Ca}_{x} \mathrm{MnO}_{3}$ system the magnetocaloric effect results for $x=0.25$ and 0.30 show a simple ferromagnetic phase transition effect. For the samples above the onset concentration for the charge ordering $(\approx 0.30)$ an anomalous magnetic entropy change is observed below the charge-ordering temperature $\left(T_{\mathrm{co}}\right)$. This effect is associated with a positive contribution from the magnetic entropy change due to charge ordering, superimposed to the negative contribution due to spin ordering. Such positive entropy contribution can be understood as the increase in accessible states due to the increase in electron mobility, under an applied magnetic field. 


\section{Acknowledgments}

The authors acknowledge FAPERJ/Brasil, FCT/Portugal (POCTI/CTM/ 35462/00 contract) and the GRICES/CAPES bilateral collaboration for financial support. J.S.A. acknowledges a scholarship from the University of Aveiro. M.S.R. also thanks CNPq for financial support.

\section{References}

[1] S.Yu. Dan'kov, A.M. Tishin, V.K. Pecharsky, K.A. Gschneidner Jr., Phys. Rev. $B$ 57, 3478 (1998).

[2] V.K. Pecharsky, K.A. Gschneidner Jr., Phys. Rev. Lett. 78, 4494 (1997).

[3] O. Tegus, E. Brück, K.H.J. Buschow, F.R. de Boer, Nature 415, 150 (2002).

[4] S. Fujieda, A. Fujita, K. Fukamichi, Appl. Phys. Lett. 81, 1276 (2002).

[5] Z.B. Guo, Y.W. Du, J.S. Zhu, H. Huang, W.P. Ding, D. Feng, Phys. Rev. Lett. 78, 1142 (1997).

[6] Colossal Magnetoresistive Oxides, Ed. Y. Tokura, Gordon and Breach, Singapore 2000.

[7] Colossal Magnetoresistance, Charge Ordering and Related Properties of Manganese Oxides, Eds. C.N.R. Rao, B. Raveau, World Scientific, Singapore 1998.

[8] M.W. Zemansky, Heat and Thermodynamics, 5th ed., McGraw-Hill, Kogakusha, Japan 1968.

[9] J.P. Araújo, V.S. Amaral, P.B. Tavares, A.A.C.S. Lourenço, F. Lencart-Silva, E. Alves, J.B. Sousa, J.M. Vieira, J. Magn. Magn. Mater. 226-230, 797 (2001).

[10] V.S. Amaral, J.P. Araújo, Yu.G. Pogorelov, J.B. Sousa, P.B. Tavares, J.M. Vieira, J. Magn. Magn. Mater. 242-245, 655 (2002).

[11] V.S. Amaral, J.P. Araújo, Yu.G. Pogorelov, J.M.B. Lopes dos Santos, P.B. Tavares, A.A.C.S. Lourenço, J.B. Sousa, J.M. Vieira, J. Magn. Magn. Mater. 226-230, 837 (2001).

[12] V.S. Amaral, J.P. Araújo, Yu.G. Pogorelov, J.B. Sousa, P.B. Tavares, J.M. Vieira, P.A. Algarabel, M.R. Ibarra, J. Appl. Phys. 93, 7646 (2003).

[13] D.I. Uzunov, Introduction to the Theory of Critical Phenomena, World Scientific, Singapore 1993.

[14] J.L. Alonso, L.A. Fernández, F. Guinea, V. Laliena, V. Martín-Mayor, Phys. Rev. $B$ 63, 054411 (2001)

[15] N.A. de Oliveira, P.J. von Ranke, M.V. Tovar Costa, A. Troper, Phys. Rev. B 66, 094402 (2002).

[16] A. Fujita, S. Fujieda, Y. Hasegawa, K. Fukamichi, Phys. Rev. B 67, 104416 (2003).

[17] Y. Tomioka, A. Asamitsu, H. Kuwahara, Y. Moritomo, Y. Tokura, Phys. Rev. B 53, R1689 (1996).

[18] V. Hardy, A. Wahl, C. Martin, Ch. Simon, Phys. Rev. B 63, 224403 (2001).

[19] P. Chen, Y.W. Du, G. Ni, Europhys. Lett. 52, 589 (2000).

[20] P. Sande, L.E. Hueso, D.R. Miguéns, J. Rivas, F. Rivadulla, M.A. López-Quintela, Appl. Phys. Lett. 79, 2040 (2001). 